\title{
Hormone receptor status does not alter the effect of trastuzumab in breast cancer
}

\author{
Tanja Ignatov', Holm Eggemann', Elke Burger², Serban Dan Costa' and \\ Atanas Ignatov ${ }^{1}$
}

1Department of Obstetrics and Gynecology, Otto-von-Guericke University, Magdeburg, Germany, ${ }^{2}$ Institute of Biometry and Medical Informatics, Otto-von-Guericke University, Magdeburg, Germany
Correspondence should be addressed to A Ignatov Email atanas.ignatov@gmail.com

\begin{abstract}
Overexpression of human epidermal growth factor receptor 2 (HER2) predicts response to anti-HER2 therapy in breast cancer. We investigated whether hormone receptor (HR) status influences the treatment benefit of trastuzumab in patients with breast cancer. Data from 8338 patients with primary nonmetastatic breast cancer from the cancer registry of Saxony-Anhalt Germany were analyzed. A total of 5554 patients were eligible for analysis. The median follow-up of the study was 6 years. Of the 5554 patients investigated, $1128(20.3 \%)$ showed HER2 overexpression and $656(58.2 \%)$ of them received adjuvant trastuzumab. The 10-year overall survival (OS) in the study cohort according to HR, HER2 status, and trastuzumab treatment was as follows: $78.4 \%$ for HR-/HER2-, 85.0\% for HR+/HER2-, 70.4\% HR-/HER2+/TRA-, 71.4\% for HR+/HER2+/TRA-, $80.9 \%$ for HR-/HER2+/TRA+, and $89.2 \%$ for HR+/HER2+/TRA+. Trastuzumab treatment improved OS in the HR- patients only in the first 3 years, whereas in the HR+ group the effect of trastuzumab was still apparent 5 years after diagnosis. Notably, the relative improvement in a patient outcome was higher for HR+ patients. Nevertheless, matching for age, histological type, tumor stage, tumor grade, and performance status between patients with HR- and HR + tumors demonstrated that the survival effect of trastuzumab was not affected by HR status; $P=0.890$. Trastuzumab treatment improves patients' survival regardless of HR status and should be offered to all HER2+ patients.
\end{abstract}

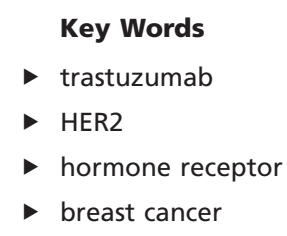

Endocrine-Related Cancer (2016) 23, 349-355

\section{Introduction}

Hormone receptor (HR) status and the expression of human epidermal growth factor receptor 2 (HER2) are two of the most important factors used for the categorization of breast cancer (Goldhirsch et al. 2013). They are prognosticators for survival and are predictive for response to endocrine and anti-HER2 therapy, respectively (Goldhirsch et al. 2013). Approximately half of HR+ breast cancers are also HER2+. Preclinical and clinical trials have suggested that HER2 overexpression is associated with increased resistance to hormonal therapy and, vice versa; HR expression is associated with a decline in the efficacy of trastuzumab (Prat \& Baselga 2008). One of the most discussed mechanisms of treatment resistance is crosstalk between HR and HER2 receptors (Osborne \& Schiff 2005). Initially, the role of HR status in trastuzumab efficacy was based on data from the neoadjuvant therapy 


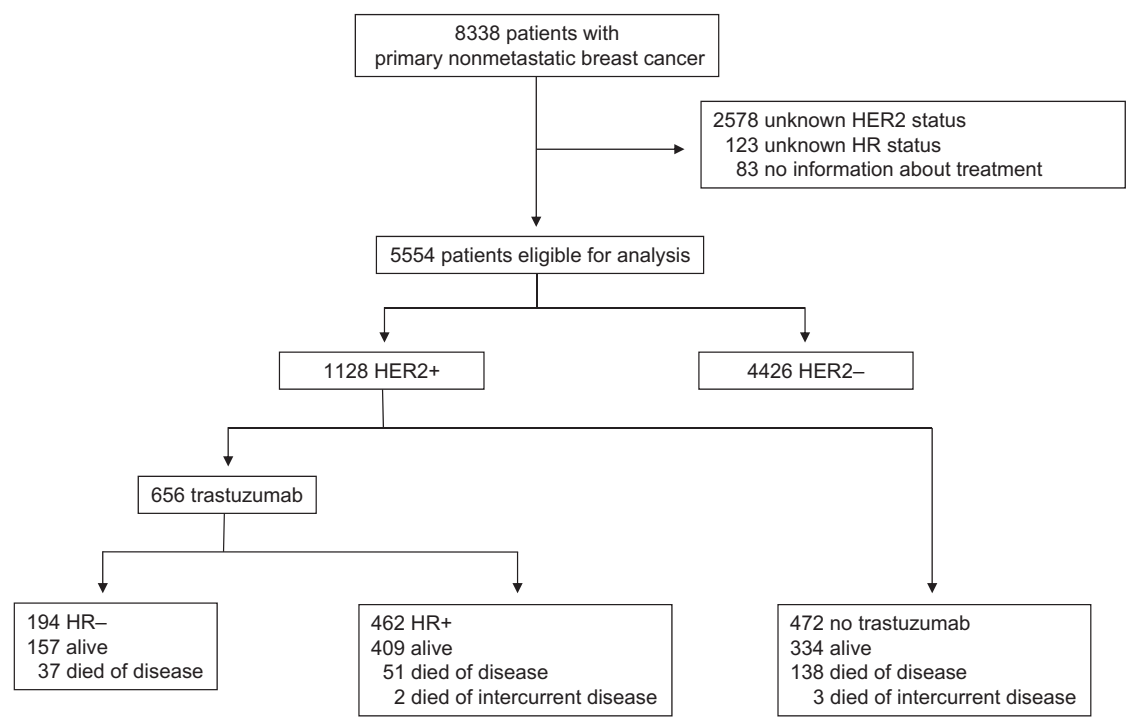

Figure 1

Study design. of HER2-positive breast cancer. Several studies have clearly demonstrated that HR expression is associated with a reduced rate of pathological complete response (pCR) (Guiu et al. 2013). However, the impact of HR status on disease-free (DFS) and overall survival (OS) of patients treated with trastuzumab is controversial (Brufsky et al. 2005, Kim et al. 2012, Vaz-Luis et al. 2012, Perez et al. 2014, Zhang et al. 2015). This controversy could be due to the heterogeneity of studies regarding adjuvant and metastatic trastuzumab treatment.

In this context, we were interested in whether HR expression affects the influence of trastuzumabbased adjuvant therapy in a large cohort of primary nonmetastatic breast cancer patients.

\section{Subjects and methods}

\section{Patients}

We investigated cases of female breast cancer included in the prospectively maintained regional cancer registry of Saxony-Anhalt, a federal state of Germany. The tumor registry contains information on diagnosis, age, tumor stage, receptor status, tumor grading, lymph node status, date of diagnosis, date of disease recurrence, date of death, and the treatment regimens used (Eggemann et al. 2015, Ignatov etal. 2015). In our cohort study, we analyzed women with primary nonmetastatic breast cancer diagnosed between 2000 and 2011 in eight hospitals in SaxonyAnhalt: University Hospital Magdeburg, Harzklinikum Dorothea Christiane Erxleben, Johanniter Clinic GenthinStendal, HELIOS Clinic Burg, and the AMEOS Clinics in Aschersleben, Halberstadt, Haldensleben, and Schönebeck.
We identified 8338 female patients with primary nonmetastatic invasive breast cancer. Of these, 2784 cases were excluded due to unknown HER2- or HR status and missing information about treatment strategy (Fig. 1). A total of 5554 eligible cases were included in the study. The follow-up ended with the patient's death, last available information in the tumor registry, or the last follow-up at 20 August 2015. This study was approved by the Research and Ethical Committee of Otto-von-Guericke University, Magdeburg, Germany. Patients gave written informed consent for data transfer to the tumor registry before treatment. Additional individual consent for this analysis was not needed. The manuscript was prepared in accordance with the Strengthening the Reporting of OBservational studies in Epidemiology statement criteria (von Elm et al. 2007).

To avoid further selection bias, a matching analysis was performed. The matching process was based on five prognostic criteria: age, tumor stage, tumor grade, lymph node status, and performance status. Age as a criterion for matching was divided into four groups: under 40, 50, 51-60, and over 61 years. The matching procedure was conducted at random and without any information about the patient outcome. The primary outcome measure was the OS, which was defined as the time from the date of diagnosis and the time of death from any cause. The OS was used as the primary outcome because information about a patient's death and its cause is automatically recorded in the cancer registry via the civil registry office (Eggemann et al. 2015, Ignatov et al. 2015), leading to a minimal loss of follow-up regarding OS, and thus keeping transfer bias to a minimum.

Published by Bioscientifica Ltd. 


\section{HER2 status and trastuzumab treatment}

The HER2 status was assessed using the HercepTest according to the manufacturer's instructions (Dako). The immunohistochemical staining of HER2 is based on staining intensity and distribution. The HER2 status was grouped as follows: 0 , no staining; $1+$, faint/barely perceptible membrane staining detected in $>10 \%$ of the tumor cells; $2+$, weak-to-moderate membrane staining $>10 \%$ of the tumor cells; and 3+, strong, circumferential membrane staining in $>30 \%$ of tumor cells. Fluorescence in situ hybridization or silver in situ hybridization testing is routinely used to assess HER2 gene amplification in cases with moderate HER2 2+ expression. Based on HER2 overexpression, the patients were divided into two main groups: HER2- and HER2+ (Fig. 1). For further analysis, they were stratified by HR status (HR- and HR+) and trastuzumab treatment (TRAand TRA+). Thus, six groups of patients were identified: HR-/HER2 - $\quad(n=639,11.5 \%), \quad$ HR+/HER $2-\quad(n=3787$, 68.2\%), HR-/HER2+/TRA- $(n=115,2.1 \%)$, HR+/HER $+/$ TRA- $(n=357,6.4 \%)$, HR-/HER2+/TRA+ $(n=194,3.5 \%)$, and HR+/HER2+/TRA+ $(n=462,8.3 \%)$.

Trastuzumab treatment was introduced in these institutions in 2003 and was used with increasing frequency in subsequent years. Most of the patients with HER2+ tumors, who did not receive trastuzumab, were diagnosed before the 'trastuzumab era' and their HER2 status was determined post factum. Between 2007 and 2011, the HER2 expression was standardized in accordance with the actual guidelines from 2007 and was done during the first diagnosis. The expression between 2000 and 2007 was performed for this analysis in accordance with the expression analysis between 2007 and 2011.

\section{Statistical analysis}

The statistical calculations were performed using SPSS Version 22.0 (SPSS). The association between HER2 status and clinical and pathological variables was assessed using the $\chi^{2}$ test or Fisher's exact test. The DFS and OS probability distribution were studied using the Kaplan-Meier method. The equality of survival curves was tested using the log rank test. DFS was defined as the time between primary diagnosis and disease recurrence of all types as well as breast cancer-specific death. OS was defined as the time between primary diagnosis and death from any cause. Univariate Cox proportional hazards regression analysis was used to identify significant prognostic factors and then the prognostic significance was evaluated using multivariate analysis. Matching between HR- and HR+ patients was done 1:1 based on patients' age, tumor stage, tumor grade, lymph node status, and performance status. The matching procedure was conducted at random and without any information about the patient outcome. The statistical analyses were two sided and $P$ values of $<0.05$ were considered to be statistically significant.

\section{Results}

Between January 2000 and December 2011, 8338 women with primary nonmetastatic invasive breast cancer underwent medical treatment in eight hospitals. After exclusion of 2784 patients, a total of 5554 with a median age of 63 years (range 24-97 years) were eligible for analysis (Fig. 1). The median follow-up time was 6 years (range 0-16 years). Patients were divided into six groups based on their HER2 and HR expression and trastuzumab treatment. Of the 5554 eligible for analysis, 1128 (20.3\%) were HER $2+$ and 656 (58.2\%) of these received trastuzumab as adjuvant therapy. One hundred and ninety-six (29.6\%) and $462(70.4 \%)$ of the trastuzumab-treated patients were HR- and HR+, respectively (Fig. 1).

Next, we wanted to compare patient outcome depending on HR status, HER2 status, and trastuzumab treatment. Among the 5554 patients eligible for analysis, 977 (17.6\%) deaths were recorded. HR negativity and HER2 positivity were associated with a poorer prognosis (Fig. 2).

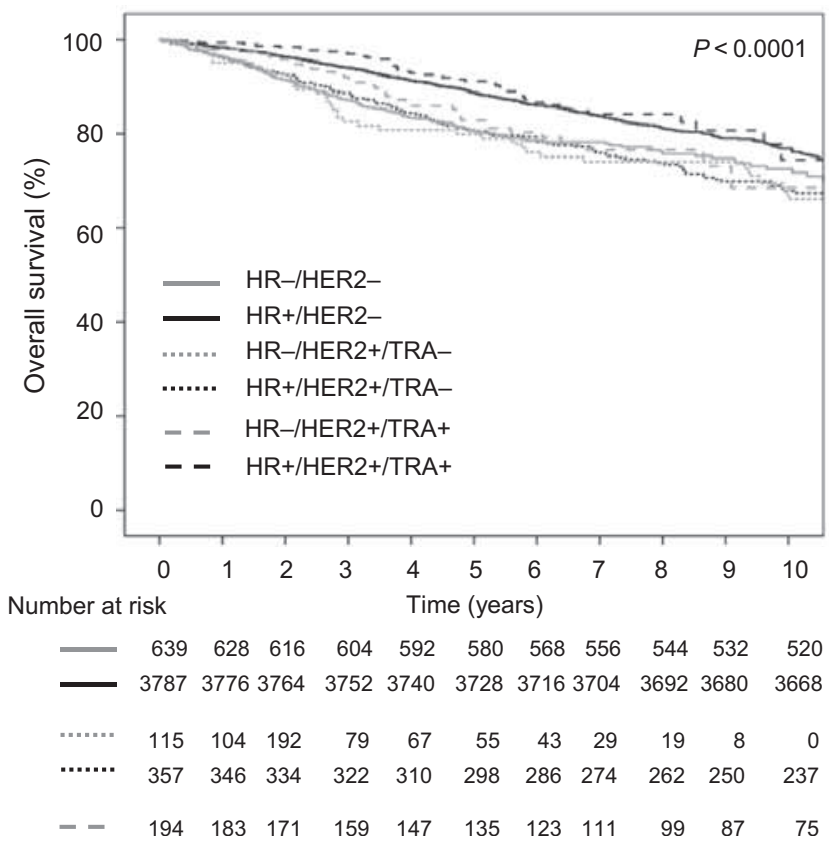

Figure 2

Kaplan-Meier analysis of overall survival for all patients. HER2, human epidermal growth factor receptor 2; HR, hormone receptor.

Published by Bioscientifica Ltd. 
Table 1 Overall survival.

\begin{tabular}{|c|c|c|c|c|c|c|c|}
\hline $\begin{array}{l}\text { Overall } \\
\text { survival }\end{array}$ & $\begin{array}{c}\text { Overall } \\
(\%)\end{array}$ & $\begin{array}{c}\text { HR-I } \\
\text { HER2- } \\
(\%)\end{array}$ & $\begin{array}{c}\text { HR+I } \\
\text { HER2- } \\
(\%)\end{array}$ & $\begin{array}{c}\text { HR-I } \\
\text { HER2+I } \\
\text { TRA- } \\
(\%)\end{array}$ & $\begin{array}{c}\text { HR+l } \\
\text { HER2+I } \\
\text { TRA- } \\
(\%)\end{array}$ & $\begin{array}{c}\text { HR-I } \\
\text { HER2+I } \\
\text { TRA+ } \\
(\%)\end{array}$ & $\begin{array}{c}\text { HR+I } \\
\text { HER2+I } \\
\text { TRA+ } \\
(\%)\end{array}$ \\
\hline 3 years & 93.0 & 87.5 & 94.2 & 82.6 & 88.3 & 92.3 & 97.6 \\
\hline 5 years & 88.4 & 82.0 & 90.0 & 80.0 & 80.7 & 85.1 & 93.1 \\
\hline 10 years & 83.3 & 78.4 & 85.0 & 70.4 & 71.4 & 80.9 & 89.2 \\
\hline
\end{tabular}

The 10-year OS of the patients based on the expression of HR and HER2 receptors and trastuzumab therapy was as follows: $78.4 \%$ for HR-/HER2-, $85.0 \%$ for HR+/ HER2-, 70.4\% for HR-/HER2+/TRA-, 71.4\% for HR+/ HER $2+/$ TRA-, $80.9 \%$ for HR-/HER2+/TRA+, and $89.2 \%$ for $\mathrm{HR}+/ \mathrm{HER} 2+/ \mathrm{TRA}+$ (Table 1). The use of trastuzumab significantly increased the 10-year OS of HER2+ breast cancer patients regardless of HR status. The relative survival effect of trastuzumab was estimated as 10.5 and $17.8 \%$ for HR- and HR+ breast cancer patients, respectively. Notably, the survival of HR+/HER2+/TRA+ patients was comparable with the survival of HR+/HER2- patients (Fig. 2). The worst prognosis was observed for HER2+ patients, who did not receive trastuzumab regardless of their HR status.

We were also interested in knowing whether the survival effect of trastuzumab is time dependent. We found that for $\mathrm{HR}+$ patients, the survival effect of trastuzumab was apparent over a period of 5 years, whereas for HR- patients, trastuzumab improved survival only for the first 3 years (Table 2). After adjustment for

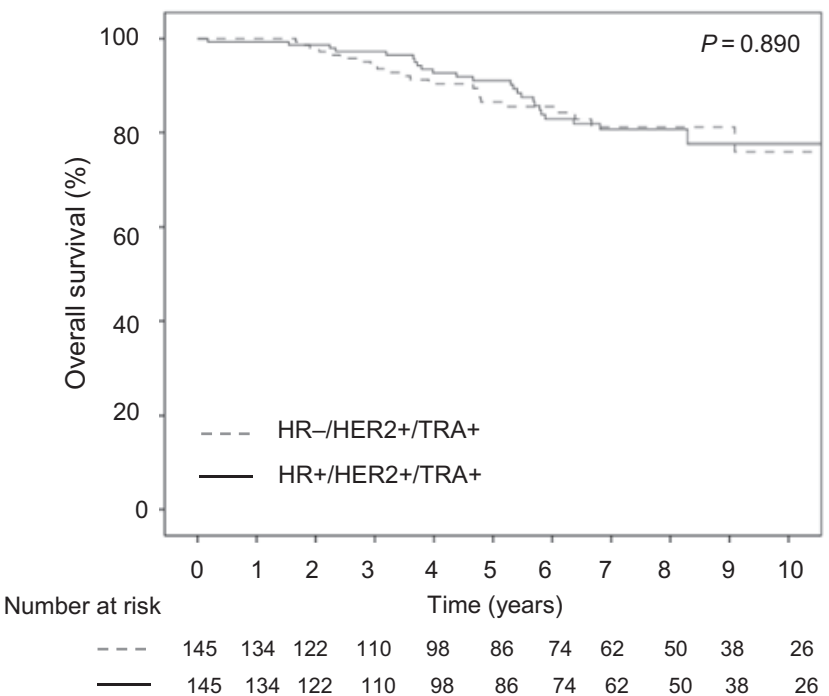

Figure 3

Kaplan-Meier analysis of overall survival for all patients after matching for age, tumor stage, histological grade, lymph node status, and performance status. HER2, human epidermal growth factor receptor 2; $\mathrm{HR}$, hormone receptor.
Table 2 Time-dependent survival effect of trastuzumab.

\begin{tabular}{|c|c|c|c|c|c|c|}
\hline \multirow[b]{2}{*}{ Characteristic } & \multicolumn{3}{|c|}{ HR-/HER2+ } & \multicolumn{3}{|c|}{ HR+/HER2+ } \\
\hline & $\begin{array}{c}\text { Hazard } \\
\text { ratio }\end{array}$ & $95 \% \mathrm{Cl}$ & $P$ value & $\begin{array}{c}\text { Hazard } \\
\text { ratio }\end{array}$ & $95 \% \mathrm{Cl}$ & $P$ value \\
\hline $0-3$ years & 0.33 & $0.14-0.79$ & 0.013 & 0.28 & $0.14-0.58$ & 0.001 \\
\hline $3-5$ years & 0.62 & $0.33-1.19$ & 0.153 & 0.51 & $0.32-0.80$ & 0.004 \\
\hline $5-10$ years & 0.80 & $0.46-1.38$ & 0.421 & 0.71 & $0.49-1.04$ & 0.080 \\
\hline
\end{tabular}

age, tumor size, lymph node status, and tumor grade, multivariate analysis revealed significantly decreased hazard due to trastuzumab treatment for HR- patients for the first 3 years, but not after 5 and 10 years (hazard ratio of death from 0 to 3 years: $0.33,95 \%$ CI: $0.14-0.79$, $P=0.013$; hazard ratio of death from 3 to 5 years: 0.62 , 95\% CI: 0.33-1.19, $P=0.153$; hazard ratio of death from 5 to 10 years: $0.80,95 \%$ CI: $0.46-1.38, P=0.421$ ). For $\mathrm{HR}+$ patients, the survival effect of trastuzumab remained favorable after adjustment for age, tumor size, lymph node status, and tumor grade during the first 5 years (hazard ratio of death from 0 to 3 years: 0.28 , 95\% CI: $0.14-0.58$, $P=0.001$; hazard ratio of death from 3 to 5 years: 0.51 , 95\% CI: 0.32-0.80, $P=0.004$; hazard ratio of death from 5 to 10 years: $0.71,95 \% \mathrm{CI}: 0.49-1.04, P=0.080$ ).

To avoid any selection bias, we analyzed the clinical and pathological characteristics of the patients with HRand HR+ breast cancer. As demonstrated in Table 3, the

Table 3 Patient and tumor characteristics of HR- and HR+ groups.

\begin{tabular}{|c|c|c|c|}
\hline Characteristic & $\begin{array}{c}\text { HER2 +/HR- } \\
n(\%)\end{array}$ & $\begin{array}{c}\text { HER2 +/HR+ } \\
n(\%)\end{array}$ & $P$ value \\
\hline Total & $194(29.6)$ & 462 (70.4) & \\
\hline Age, mean (range) & 58.75 & 56.54 & 0.031 \\
\hline \multicolumn{4}{|l|}{ Tumor size $(\mathrm{cm})$} \\
\hline$\leq 2$ & $156(87.2)$ & $384(89.5)$ & \\
\hline$>2$ & $23(12.8)$ & 45 (10.5) & 0.275 \\
\hline Missing & 15 & 33 & \\
\hline \multicolumn{4}{|l|}{ Lymph node status } \\
\hline Negative & $104(55.6)$ & 256 (57.5) & \\
\hline Positive & $83(44.4)$ & $189(42.5)$ & 0.428 \\
\hline Missing & 7 & 17 & \\
\hline \multicolumn{4}{|l|}{ Grade } \\
\hline 1 & $0(0)$ & $25(5.5)$ & \\
\hline 2 & $57(30.3)$ & $259(57.4)$ & 0.0001 \\
\hline 3 & 131 (69.7) & 167 (37.0) & \\
\hline Missing & 6 & 11 & \\
\hline $\begin{array}{l}\text { Ki-67, \% mean } \\
\text { (range) }\end{array}$ & $\begin{array}{c}54.3 \\
(3-95)\end{array}$ & $\begin{array}{c}24.7 \\
(1-95)\end{array}$ & 0.0001 \\
\hline \multicolumn{4}{|l|}{ Chemotherapy } \\
\hline Anthracycline & 38 (19.6) & 89 (19.3) & \\
\hline Taxane & $19(9.8)$ & $47(10.2)$ & 0.886 \\
\hline Both & $99(51.0)$ & $227(49.1)$ & \\
\hline Other & $2(1.0)$ & $12(2.6)$ & \\
\hline Not specified & $36(18.6)$ & 87 (18.8) & \\
\hline
\end{tabular}


basic characteristics in the HR-/HER2+ and HR+/HER2+ groups were not well balanced and the tumor and patient characteristics were unequally distributed. HR- tumors were more likely to present with poor histological grade and higher mean value of Ki-67 expression. To avoid further confounder and selection bias caused by treatment choice, we carried out matching analysis based on five prognostic criteria: patients' age, tumor stage, tumor grading, lymph node status, and performance status. The patients were matched 1:1 in accordance with their HR status. Based on this analysis, we identified 290 patients treated with trastuzumab, of whom 145 were HR- and 145 were HR+ (Fig. 3). Notably, the survival effect of trastuzumab was similar in the HR-/HER2+ and HR+/ HER2+ groups.

\section{Discussion}

In this large retrospective study with a median follow-up of 6 years, we found that adjuvant treatment of breast cancer with trastuzumab significantly increased the OS of all HER2+ patients. This effect was independent of HR status. Additionally, this trial confirms that HR positivity remains an important favorable factor even for HER2+ patients.

As the establishment of the predictive value of HER2 expression for anti-HER2 treatment, the efficacy of trastuzumab treatment in HER2+ tumors has been evaluated in different studies. It has been demonstrated that trastuzumab improves survival in all HER2+ breast cancers (Slamon et al. 2011). Randomized trials with neoadjuvant use of trastuzumab demonstrated a significant increase in the rate of pCR in HER2+ breast cancer (Guiu et al. 2013). It has been found that HRtumors are associated with a higher rate of pCR compared with HR+ tumors when treated with trastuzumab and other anti-HER2 agents (Bhargava et al. 2011, Guiu et al. 2013). Based on these data, it has been proposed that patients with HR- HER2+ disease might benefit from such therapy. It has also been suggested that the pCR rate should be used as a surrogate marker to predict patient survival and to identify those patients who could benefit from anti-HER2 therapy. However, it has recently been demonstrated that these observations are only valid for patients with luminal B/HER2-, HER2+ (nonluminal), and triple-negative breast cancer, and not for those with luminal B/HER2+ or luminal A tumors (von Minckwitz et al. 2012). Nevertheless, the impact of HR status on trastuzumab efficacy in terms of patients' survival has been poorly investigated. The available data are very discrepant. Most of the data were obtained from trials on metastatic breast cancer. Similar to the data obtained in neoadjuvant trials and in contrast to our findings, Montemurro et al. (2012) and Zhang et al. (2015) found that HR negativity is associated with an increased survival benefit of trastuzumab. Montemuro and coworkers showed that the estrogen receptor (ER) positivity was associated with reduced overall response rate to firstline trastuzumab and chemotherapy, but with improved progression-free survival when endocrine therapy was added to maintenance trastuzumab in responding patients. Very recently, similar results were published by Vici et al. (2016), demonstrating that trastuzumab improved relapse-free survival and breast cancer-specific survival in all patients, but the effect in tumors expressing both HR and ER was not significant. In contrast, Dawood et al. (2010) found that HR positivity was associated with improved survival in patients with metastatic breast cancer. Similar to this study, they found that HR status was an important prognostic factor. A recent retrospective analysis of three prospective trials suggested that HR did not affect the benefit from trastuzumab for metastatic breast cancer (Brufsky et al. 2005) and was consistent with our observation. However, any comparison between our results and such trials in metastatic disease should be interpreted with caution given the differences in patients' cohorts, including treatment characteristics and the primary outcomes used in adjuvant and metastatic settings. Nevertheless, all these data demonstrate that dual blockade of the HR and HER2 signaling pathway might be indicated for HR+ and HER2+ breast cancer (Cortes \& Baselga 2009).

To the best of our knowledge, the impact of HR status on the survival effect of trastuzumab in early breast cancer has only been investigated in one study (Vaz-Luis et al. 2012). In this study of 3394 patients with HER2+ breast cancer, the trastuzumab survival effect was significantly affected by HR status. HR positivity was associated with a favorable DFS and OS. The discrepancy with our data could be attributed to the unbalanced groups in the study by Vaz-Luis et al. (2012). Similar to our collective, HR- tumors were more likely to present unfavorable prognostic factors such as a higher histological grade. To exclude possible selection bias, we performed a matching analysis for these factors, but this was not performed in the aforementioned study. In this context, we found that patients with $\mathrm{HR}-$ and $\mathrm{HR}+$ tumors benefit from adjuvant treatment with trastuzumab to a similar order of magnitude. However, similar to this study, Vaz-Luis and coworkers found that patients with HR- tumors had an

Published by Bioscientifica Ltd. 
increased risk of late tumor death and that HR expression was a favorable prognostic factor in HER2+ tumors.

Among all prospective clinical trials evaluating the clinical effect of adjuvant treatment with trastuzumab, only one study investigated the influence of HR status on its efficacy (Perez et al. 2014). In this trial, the trastuzumab effect was not significantly influenced by HR status. The 10-year OS rates for patients with HR- disease receiving adjuvant trastuzumab and chemotherapy or only chemotherapy were 81.6 and $73.0 \%$, respectively, and 86.0 and $77.1 \%$ for patients with $\mathrm{HR}+$ breast cancer, who received trastuzumab or did not receive trastuzumab as adjuvant therapy (Perez et al. 2014). These data are consistent with our observations. We observed a significant improvement of OS after trastuzumab in both HR- and HR+ patients. In our cohort, the 10-year OS rates for patients with HRdisease who did or did not receive adjuvant trastuzumab were 80.9 and $70.4 \%$, respectively, and 89.2 and $71.4 \%$ for patients with HR+ breast cancer, who did or did not receive trastuzumab as adjuvant therapy.

As mentioned above, in HR- patients we found that the trastuzumab survival effect was significant only in the first 3 years beyond diagnosis, whereas the effect of trastuzumab on OS in the HR+ group persisted for 5 years after diagnosis. This is consistent with several prior studies describing different rates of recurrence and hazard of death depending on HR status (Dignam et al. 2009, Early Breast Cancer Trialists' Collaborative Group et al. 2011, Vaz-Luis et al. 2012, Strasser-Weippl et al. 2015) suggesting that HR- and HR+ HER2+ breast cancer represent two different entities (Burstein 2005). In this regard, our observations highlight the fact that HR status remains an important prognostic factor regardless of HER 2 status and trastuzumab treatment.

One limitation of this study is that the DFS and rate of recurrence were not investigated. Other limitations of this study include: (i) its retrospective character with all associated bias and (ii) the lack of central pathological review regarding HR and HER2 status.

The strengths of this study are: (i) large sample size with long follow-up; (ii) matching analysis between HRand $\mathrm{HR}+$ patients regarding the most important prognostic factors; (iii) high level of external validity as trastuzumab treatment was investigated under real clinical conditions (e.g. multicentric, the study population was similar to the general population, and the exclusion criteria were kept to a minimum); (iv) the population-based character of the cohort ruled out referral bias; (v) minimal loss to follow-up regarding OS; and (vi) trastuzumab treatment was performed in a standardized manner in all centers, thus excluding performance bias.

In conclusion, this study demonstrates that trastuzumab improved the OS of patients with HER2+ breast cancer. This survival effect is independent of HR status. Our data provide compelling evidence that trastuzumab should be considered as a standard treatment for all HER2+ breast cancers.

\section{Declaration of interest}

The authors declare that there is no conflict of interest that could be perceived as prejudicing the impartiality of the research reported.

\section{Funding}

This research did not receive any specific grant from any funding agency in the public, commercial, or not-for-profit sector.

\section{References}

Bhargava R, Dabbs DJ, Beriwal S, Yildiz IA, Badve P, Soran A, Johnson RR, Brufsky AM, Lembersky BC, McGuire KP, et al. 2011 Semiquantitative hormone receptor level influences response to trastuzumab-containing neoadjuvant chemotherapy in HER2positive breast cancer. Modern Pathology 24 367-374. (doi:10.1038/ modpathol.2010.209)

Brufsky A, Lembersky B, Schiffman K, Lieberman G \& Paton VE 2005 Hormone receptor status does not affect the clinical benefit of trastuzumab therapy for patients with metastatic breast cancer. Clinical Breast Cancer 6 247-252. (doi:10.3816/cbc.2005.n.027)

Burstein HJ 2005 The distinctive nature of HER2-positive breast cancers. New England Journal of Medicine 353 1652-1654. (doi:10.1056/ nejmp058197)

Cortes J \& Baselga J 2009 How to treat hormone receptor-positive, human epidermal growth factor receptor 2-amplified breast cancer. Journal of Clinical Oncology 27 5492-5494. (doi:10.1200/ jco.2009.23.8089)

Dawood S, Broglio K, Buzdar AU, Hortobagyi GN \& Giordano SH 2010 Prognosis of women with metastatic breast cancer by HER2 status and trastuzumab treatment: an institutional-based review. Journal of Clinical Oncology 28 92-98. (doi:10.1200/jco.2008.19.9844)

Dignam JJ, Dukic V, Anderson SJ, Mamounas EP, Wickerham DL \& Wolmark N 2009 Hazard of recurrence and adjuvant treatment effects over time in lymph node-negative breast cancer. Breast Cancer Research and Treatment 116 595-602. (doi:10.1007/s10549. 008-0200-5)

Early Breast Cancer Trialists' Collaborative Group, Davies C, Godwin J, Gray R, Clarke M, Cutter D, Darby S, McGale P, Pan HC, Taylor C, et al. 2011 Relevance of breast cancer hormone receptors and other factors to the efficacy of adjuvant tamoxifen: patient-level metaanalysis of randomised trials. Lancet 378 771-784. (doi:10.1016/ s0140-6736(11)60993-8)

Eggemann H, Ignatov T, Burger E, Kantelhardt EJ, Fettke F, Thomssen C, Costa SD \& Ignatov A 2015 Moderate HER2 expression as a prognostic factor in hormone receptor positive breast cancer. Endocrine-Related Cancer 22 725-733. (doi:10.1530/ERC-15-0335)

Goldhirsch A, Winer EP, Coates AS, Gelber RD, Piccart-Gebhart M, Thurlimann B, Senn HJ \& Panel M 2013 Personalizing the treatment of women with early breast cancer: highlights of the
(C) 2016 Society for Endocrinology Printed in Great Britain
Published by Bioscientifica Ltd 
St Gallen International Expert Consensus on the Primary Therapy of Early Breast Cancer 2013. Annals of Oncology 24 2206-2223. (doi:10.1093/annonc/mdt303)

Guiu S, Mouret Reynier MA, Toure M \& Coudert B 2013 Predictive factors of response in HER2-positive breast cancer treated by neoadjuvant therapy. Journal of Oncology 2013 article 854121. (doi:10.1155/2013/854121)

Ignatov T, Eggemann H, Burger E, Fettke F, Costa SD \& Ignatov A 2015 Moderate level of HER2 expression and its prognostic significance in breast cancer with intermediate grade. Breast Cancer Research and Treatment 151 357-364. (doi:10.1007/s10549-0153407-2)

Kim MM, Dawood S, Allen P, Sahin AA, Woodward WA, Smith BD, Strom EA, Hunt KK, Meric-Bernstam F, Gonzalez-Angulo AM, et al. 2012 Hormone receptor status influences the locoregional benefit of trastuzumab in patients with nonmetastatic breast cancer. Cancer 118 4936-4943. (doi:10.1002/cncr.27502)

Montemurro F, Rossi V, Cossu Rocca M, Martinello R, Verri E, Redana S, Adamoli L, Valabrega G, Sapino A, Aglietta M, et al. 2012 Hormonereceptor expression and activity of trastuzumab with chemotherapy in HER2-positive advanced breast cancer patients. Cancer 118 17-26. (doi:10.1002/cncr.26162)

Osborne CK \& Schiff R 2005 Estrogen-receptor biology: continuing progress and therapeutic implications. Journal of Clinical Oncology 23 1616-1622. (doi:10.1200/jco.2005.10.036)

Perez EA, Romond EH, Suman VJ, Jeong JH, Sledge G, Geyer CE Jr, Martino S, Rastogi P, Gralow J, Swain SM, et al. 2014 Trastuzumab plus adjuvant chemotherapy for human epidermal growth factor receptor 2-positive breast cancer: planned joint analysis of overall survival from NSABP B-31 and NCCTG N9831. Journal of Clinical Oncology 32 3744-3752. (doi:10.1200/jco.2014.55.5730)

Prat A \& Baselga J 2008 The role of hormonal therapy in the management of hormonal-receptor-positive breast cancer with co-expression of HER2. Nature Clinical Practice Oncology 5 531-542. (doi:10.1038/ncponc1179)
Slamon D, Eiermann W, Robert N, Pienkowski T, Martin M, Press M, Mackey J, Glaspy J, Chan A, Pawlicki M, et al. 2011 Adjuvant trastuzumab in HER2-positive breast cancer. New England Journal of Medicine 365 1273-1283. (doi:10.1056/nejmoa0910383)

Strasser-Weippl K, Horick N, Smith IE, O'Shaughnessy J, Ejlertsen B, Boyle F, Buzdar AU, Fumoleau P, Gradishar W, Martin M, et al. 2015 Long-term hazard of recurrence in HER2+ breast cancer patients untreated with anti-HER2 therapy. Breast Cancer Research 1756. (doi:10.1186/s13058-015-0568-1)

Vaz-Luis I, Ottesen RA, Hughes ME, Marcom PK, Moy B, Rugo HS, Theriault RL, Wilson J, Niland JC, Weeks JC, et al. 2012 Impact of hormone receptor status on patterns of recurrence and clinical outcomes among patients with human epidermal growth factor-2positive breast cancer in the National Comprehensive Cancer Network: a prospective cohort study. Breast Cancer Research 14 R129. (doi:10.1186/bcr3324)

Vici P, Pizzuti L, Sperduti I, Frassoldati A, Natoli C, Gamucci T, Tomao S, Michelotti A, Moscetti L, Gori S, et al. 2016 "Triple positive" early breast cancer: an observational multicenter retrospective analysis of outcome. Oncotarget [in press]. (doi:10.18632/oncotarget.7480)

von Elm E, Altman DG, Egger M, Pocock SJ, Gotzsche PC, Vandenbroucke JP for the STROBE Initiative 2007 The Strengthening the Reporting of Observational Studies in Epidemiology (STROBE) statement: guidelines for reporting observational studies. Lancet $\mathbf{3 7 0}$ 1453-1457. (doi:10.1016/s0140-6736(07)61602-x)

von Minckwitz G, Untch M, Blohmer JU, Costa SD, Eidtmann H, Fasching PA, Gerber B, Eiermann W, Hilfrich J, Huober J, et al. 2012 Definition and impact of pathologic complete response on prognosis after neoadjuvant chemotherapy in various intrinsic breast cancer subtypes. Journal of Clinical Oncology 30 1796-1804. (doi:10.1200/ jco.2011.38.8595)

Zhang L, Li J, Xiao Y, Cui H, Du G, Wang Y, Li Z, Wu T, Li X \& Tian J 2015 Identifying ultrasound and clinical features of breast cancer molecular subtypes by ensemble decision. Science Reports 5 11085. (doi:10.1038/srep11085)

Received in final form 16 March 2016

Accepted 23 March 2016

Accepted Preprint published online 23 March 2016
๑ 2016 Society for Endocrinology Printed in Great Britain
Published by Bioscientifica Ltd 